\title{
REVERSE ENGINEERING AND PROCESS MODELING OF GEOMETRICALLY COMPLEX STIFFNED SHELL STRUCTURES
}

\author{
Cyprien Wolff ${ }^{1}$, Jean-Louis Batoz ${ }^{2}$, Arnaud Delameziere ${ }^{1}$ and Eduard Antaluca ${ }^{2}$ \\ ${ }^{1}$ Université de Lorraine - CNRS - Mines Nancy, Laboratoire d'Etude des Microstructures et de \\ Mécanique des Matériaux, LEM3 UMR CNRS 7239, GIP-InSIC \\ 88100 Saint-Dié-des-Vosges, France \\ cyprien.wolff@univ-lorraine.fr; arnaud.delameziere@univ-lorraine.fr \\ and http://www.lem3.univ-lorraine.fr \\ ${ }^{2}$ Alliance Sorbonne Université-Université de Technologie de Compiègne, UMR 7337 et EA 7284 , \\ 60200 Compiègne, \\ batoz@utc.fr, eduard.antaluca@utc.fr
}

Key words: Shell Structure, Shell Finite Elements, Reverse Engineering, 3D Modelling.

\begin{abstract}
Thin-walled stiffened shell structures are very common as industrial products and their analyses by the finite element method can be difficult, in particular when the part is geometrically complex as found in the field of packaging. When the initial CAD definition is not available, reverse engineering is necessary, often based on the use of $3 D$ scanners, in order to obtain a performing $3 D$ geometrical model before considering finite element computations. The process of data capture to FEM can be time consuming and difficult for parts with several $3 D$ stiffeners, depending on the strategies to reconstruct the part. This work focuses on the evaluation of three strategies applied to quite simple thin-walled parts using Geomagic and Abaqus software for the reconstruction and the simulation, respectively. Regarding FE simulations we focus on the computations of the first free frequency since this situation offers interesting comparison with experimental results. The criteria for the evaluation of the strategy are the times for scanning and processing of the data, the reconstruction, of the geometry for the FEM computations. We also study the influence of the strategies on the results of the simulations. These results depend on the type of element used and the present study reveals that for certain strategies the choice is restricted to the use of solid (mainly tetrahedron) elements, while another strategy allows the use of shell or solidshell elements. In this that case only one element through the thickness is used leading to very acceptable results for reduced calculation times but for a longer backward reconstruction time. The advantages and drawbacks of the $3 D$ geometry reconstruction combined with FEM computational strategies are discussed.
\end{abstract}

\section{INTRODUCTION}

Thin-walled structures are very common in sheet metal manufacturing, injected parts, etc. Their structural analysis by the Finite Elements Method (FEM) requires geometry in the 
digital format which does not always exist. 3D scanner is a solution to obtain a digital model from a real object. Nevertheless, the geometrical information in the form of point cloud or a 3D mesh cannot be directly used in FEM software without a specific processing.

Different processes from digitization to simulation have been proposed in the literature, in civil engineering, medical field, mechanical engineering [1-3]. The thin-walled parts can be reconstructed by using different strategies depending on the geometrical complexity of their structure. Sometimes, the reconstruction phase can disappear. For example, Schneider et al. [4] obtained a good quality 3D mesh generated after the scanning phase that can be directly converted into a mesh of triangular shell elements. Gentilini et al. [5] used the bubble packing algorithm to transform a dense digitized mesh created into a mesh suitable for FEM analysis. Recently, Greco et al. [6] proposed a reconstruction by fitting a B-spline surface on the point cloud of a deep drawn component. The basic functions describing this surface are also used in the simulation to avoid the classical discretization of the structure with shell elements. This promising method is limited to simple geometries which can be approximate by a unique smooth surface. However, most of the previous strategies are not yet available in commercial software dedicated to Reverse Engineering (RE) and cannot be easily used by a design engineer. Nevertheless, the number of methods and tools offered in most of commercial RE software is increasing and therefore it is necessary to help the designers to choose the most appropriate methodology to reconstruct and simulate complex stiffened shell parts with a desired accuracy and within a reasonable CPU time
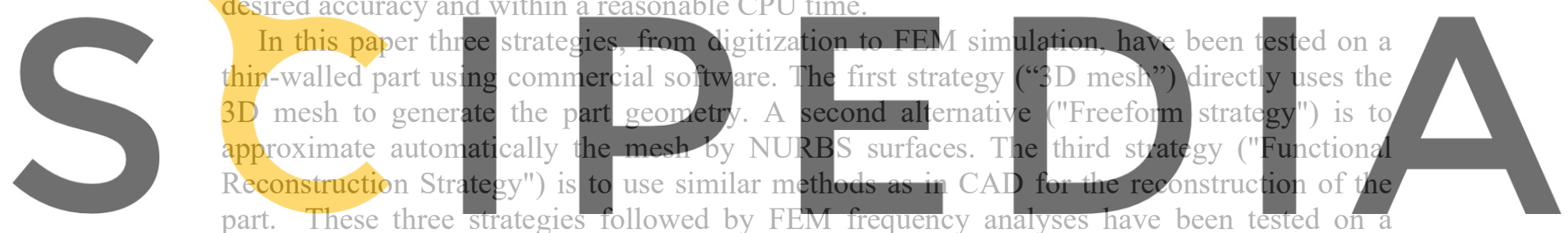

part. These three strategies followed by FEM frequency analyses have been tested on a Register for ffreen.at https//www.scipedia.com to download the version without the watermark

\section{THREE STRATEGIES FROM DIGITIZATION TO FEM SIMULATION}

Most of investigations in the field of RE [7-9], are proposing a process divided into four steps namely, 1) Data capture, 2) Pre-processing, 3) Segmentation and region classification, 4) Reconstruction. The three strategies defined in the introduction have been tested by following these different steps on a simple part which is a square plate $(200 \times 200 \mathrm{~mm})$ strengthened by stiffeners on one side (see Figure 1). The eight stiffeners are arranged to form a grid (height and thickness of the stiffeners: $15 \mathrm{~mm}$ and $3 \mathrm{~mm}$, respectively). 

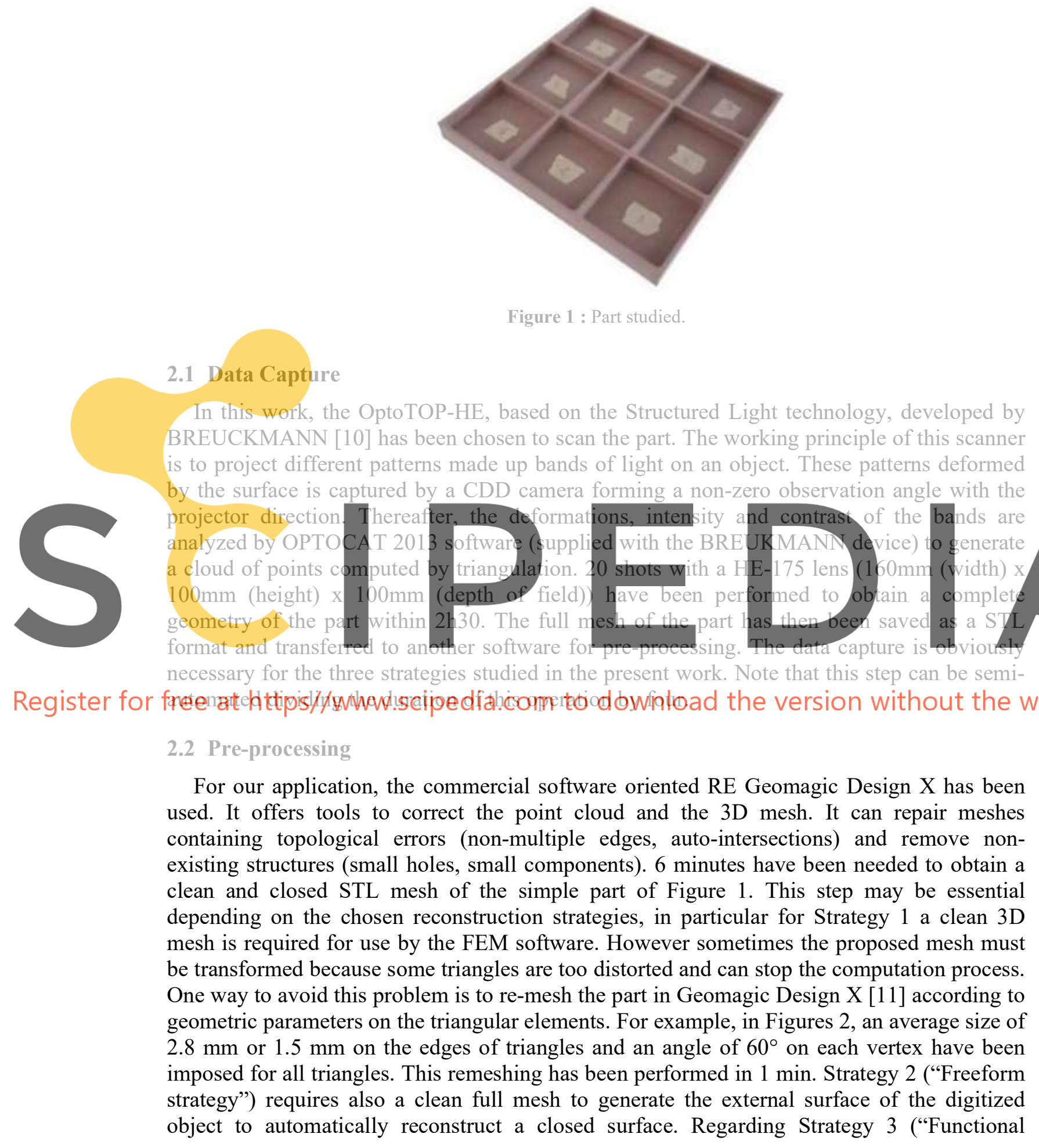

Figure 1 : Part studied.

.1. Data Capture

In this work, the OptoTOP-HE, based on the Structured Light technology, developed by BREUCKMANN [10] has been chosen to scan the part. The working principle of this scanner is to project different patterns made up bands of light on an object. These patterns deformed by the surface is captured by a CDD camera forming a non-zero observation angle with the projector direction. Thereafter, the deformations, intensity and contrast of the bands are
analyzed by OPTOCAT 2013 software (supplied with the BREUKMANN device) to generate
a cloud of points computed by triangulation. 20 shots with a HE-175 lens (160mm (width) x
$100 \mathrm{~mm}$ (height) x $100 \mathrm{~mm}$ (depth of field)) have been performed to obtain a complete
geometry of the part within $2 \mathrm{~h} 30$. The full mesh of the part has then been saved as a STL
format and transfered to another software for pre-processing. The data capture is obviousiy
necessary for the three strategies studied in the present work. Note that this step can be semi-

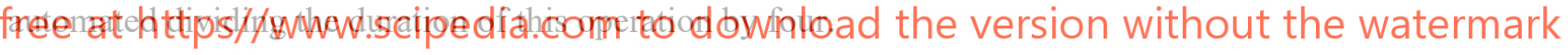

For our application, the commercial software oriented RE Geomagic Design X has been used. It offers tools to correct the point cloud and the 3D mesh. It can repair meshes containing topological errors (non-multiple edges, auto-intersections) and remove nonexisting structures (small holes, small components). 6 minutes have been needed to obtain a clean and closed STL mesh of the simple part of Figure 1. This step may be essential depending on the chosen reconstruction strategies, in particular for Strategy 1 a clean 3D mesh is required for use by the FEM software. However sometimes the proposed mesh must be transformed because some triangles are too distorted and can stop the computation process. One way to avoid this problem is to re-mesh the part in Geomagic Design X [11] according to $2.8 \mathrm{~mm}$ or $1.5 \mathrm{~mm}$ on the edges of triangles and an angle of $60^{\circ}$ on each vertex have been imposed for all triangles. This remeshing has been performed in 1 min. Strategy 2 ("Freeform object to automatically reconstruct a closed surface. Regarding Strategy 3 ("Functional 
Reconstruction Strategy"), if the functional surfaces necessary for the reconstruction of the part are relatively clean, a pre-processing is not needed as it will be shown later.

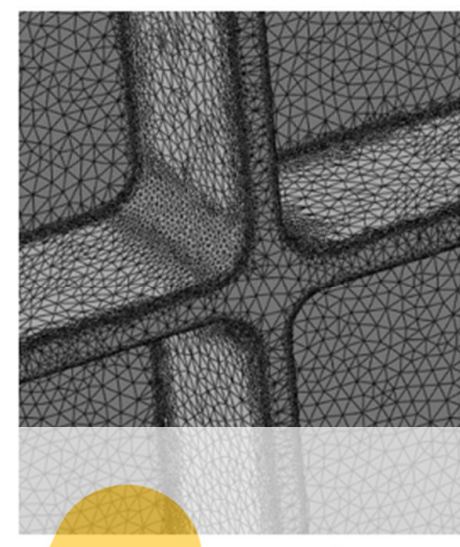

a)

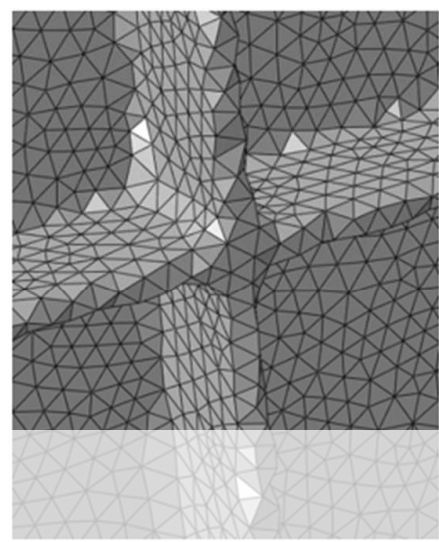

b)

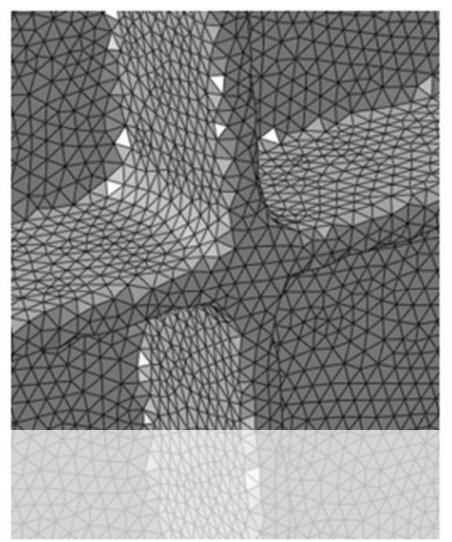

c)

Figure $2:$ a) Zoom on the original mesh. Regular remesh obtained with different element size b) $2.8 \mathrm{~mm}, \mathrm{c})$ $1.5 \mathrm{~mm}$, in Geomagic Design X software.

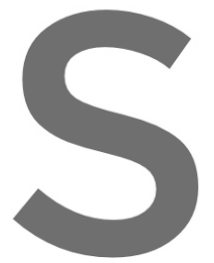

\subsection{Segmentation an \\ The simple part reconstructed by Strategy software can recogniz into cells consistent}
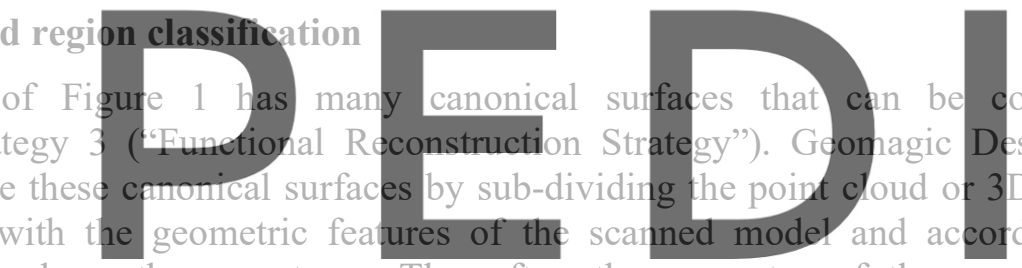

geometrical criteria such as the curvatures. Thereafter, the geometry of these cells are identified, if possible, with canonical forms (plane, cylinder, ...) or as a free form. Note that

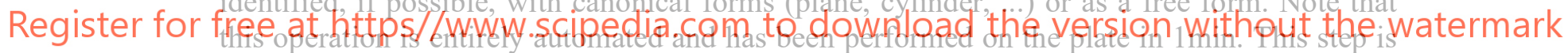
not necessary for Strategies 1 and 2 because they do not need shape recognition to generate geometries. It is also interesting to use the segmentation and region classification for the alignment of the digitized part on the axis system of the software. 30 seconds has been required to perform this operation.

\subsection{Reconstruction}

Reconstruction is undoubtedly the most complicated step of the RE process because it consists of different reconstruction strategies which can be applied on the same part. In the case of Strategy 2 ("Freeform strategy"), the point cloud or the 3D mesh is divided into patches In Geomagic Design $\mathrm{X}$, the patch areas are delimited by curves automatically extracted from the strong curvatures of the part (see, Figure 3a). However, if the results obtained by this automatic process are not satisfactory, it is possible to add new curves- The patches can be generated automatically without these curves [12]. This approach offers a deviation error between the adjusted surfaces and the digitized data smaller than the manual method, such as shown on Figure 3c where the deviation is in the order of $+-0.1 \mathrm{~mm}$. However, patches are unstructured and can lead to distortion of elements during the meshing 
process. Once the patches have been created, a volume has been generated by filling the closed surface and saved as STEP format.

Patches structured by the user Patches generated automatically

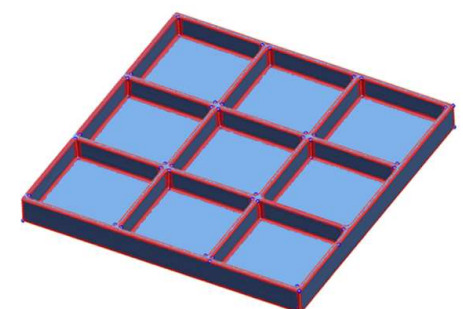

a) Grid of curves performed Deviation $(\mathrm{mm})$
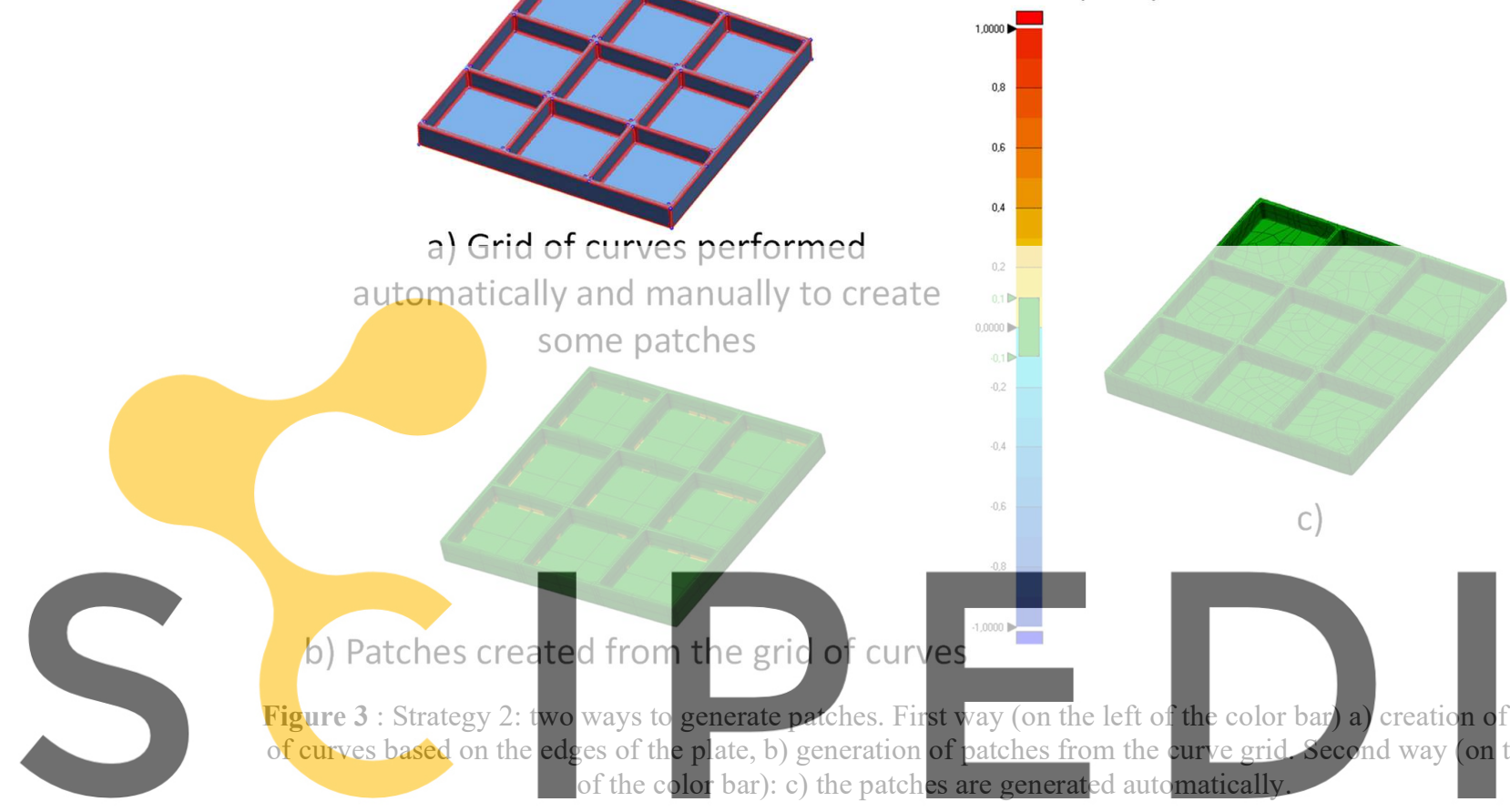

c)

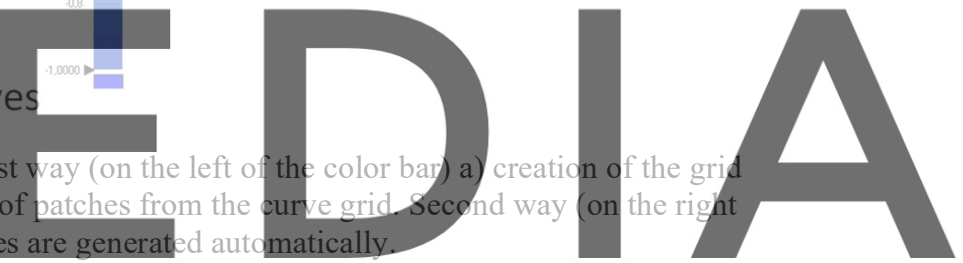

For strategy 3 ("functional reconstruction strategy"), a complete and clean mesh is not

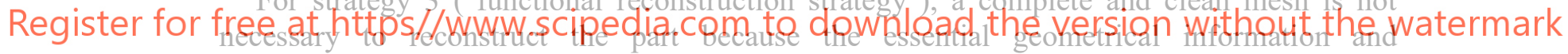
dimensions are available on the mesh. In the case of the plate, a partial mesh, illustrated in Figure 4, is sufficient for reconstruction because its volume can be defined. In addition, it contains a square pattern formed by the stiffeners which will be repeated during the reconstruction. This mesh consists of 6 views reducing the time needed for the data capture step which is estimated at $21 \mathrm{~min}$. The reconstruction of the plate is based on 3 parallel planes created from the segmentation results. They respectively delimit the bottom, the thickness and the top of the plate. Then, a cross section, parallel to the previous planes and located at the mid-height of the piece, cuts the mesh to extract the edges of the plate and the square cavity. Each edge is projected separately onto the first two planes to create two independent sketches. These two sketches are automatically geometrically constrained (perpendicularly, tangentially, ...) by Geomagic Design X. Then, dimensional constraints are added by the user. Volumes are generated by extrusion from these sketches. A small parallelepiped is created, repeated, subtracted from the volume of the plate by a Boolean operation to create nine cavities (see figure 4). The final volume is saved in a STEP format. The time spent to execute this operation is $12 \mathrm{~min}$. 


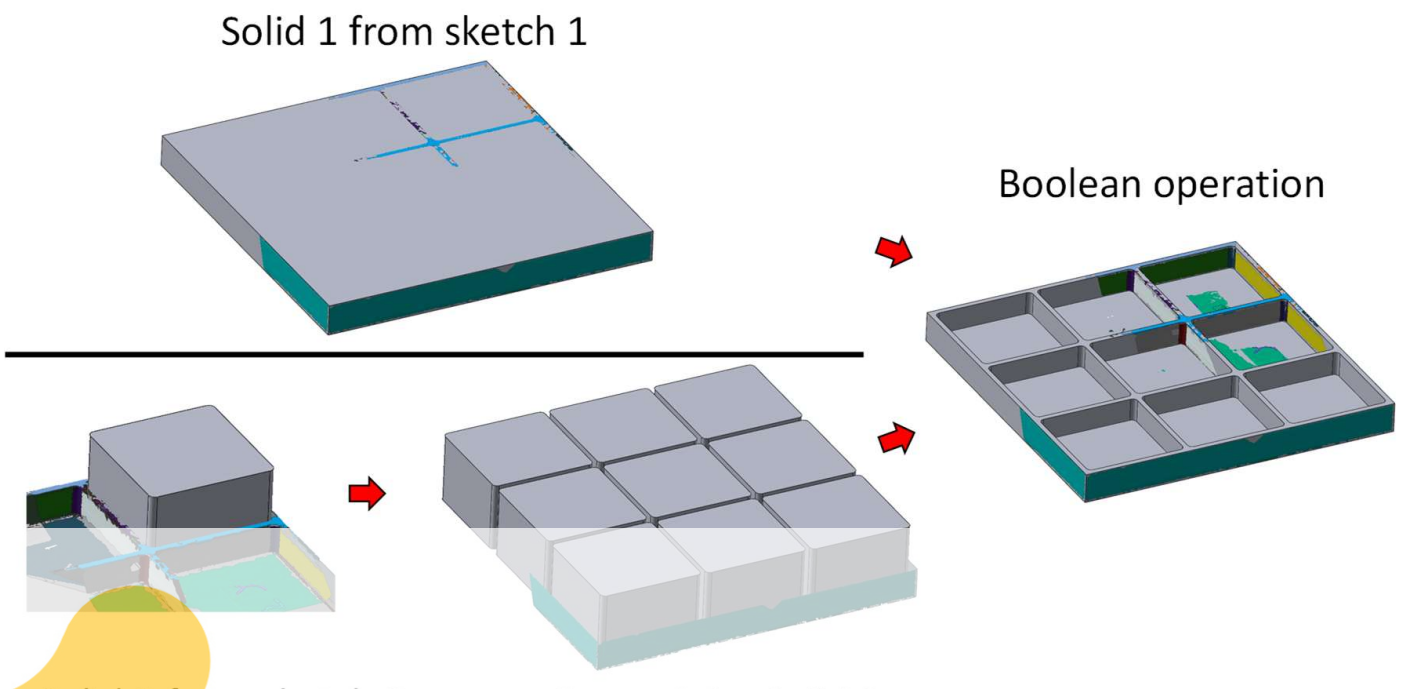

Solid 2 from sketch 2

\section{Copy of the Solid 2 \\ according to two \\ directions}
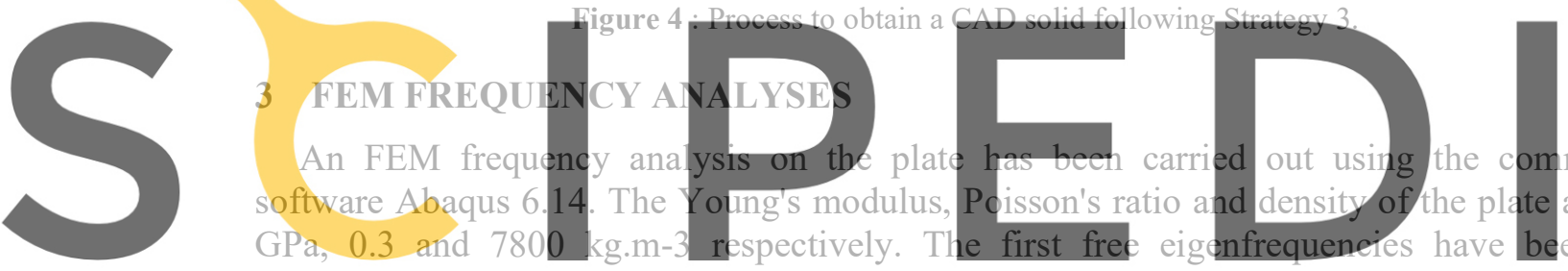

$\mathrm{GPa}, 0.3$ and $7800 \mathrm{~kg} \cdot \mathrm{m}-3$ respectively. T

to obtain reference values of the

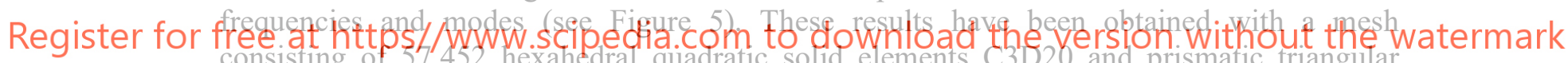
consisting of 57,452 hexahedral quadratic solid elements C3D20 and prismatic triangular solid elements C3D15 with a size of $2 \mathrm{~mm}$, (3 elements in thickness and 819,255 degrees of freedom).

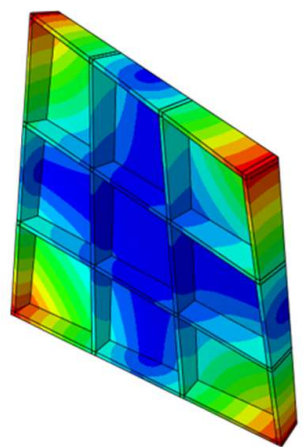

Figure $5: 1^{\text {st }}$ mode at $262 \mathrm{~Hz}$ computed using the original CAD of the plate for a fine mesh consisting of 57,452 quadratic $\mathrm{H} 8 \mathrm{C} 3 \mathrm{D} 20$ and triangular prismatic C3D15 elements (determined after a convergence study). 
The geometries of Strategy 1 have been generated in Abaqus from imported STL meshes using a plug-in called "Create a geometry from a mesh" to be added to Abaqus. The two STL meshes with average triangular sizes of $2.8 \mathrm{~mm}$ and $1.5 \mathrm{~mm}$ have been subjected to this transformation within $2 \mathrm{~min}$ and $10 \mathrm{~min}$, respectively. Linear (C3D4) or quadratic (C3D10) tetrahedral elements have been used because they can discretize all the geometries. The average size of the FEM elements is equal to the size of the triangles of the STL mesh. The geometry of strategy 2 is also limited to tetrahedral elements because of the smooth edges produced by the patches. To compare these two strategies, the size and types of elements of Strategy 1 have also been applied for Strategy 2.

Figure 6 shows the first frequency calculated from different meshes as a function of the number of degrees of freedom. The frequency calculated from linear elements (C3D4) decreases as the mesh becomes more refined. For instance, for Strategy 1, the frequency overestimates the reference value by $70 \%$ for a mesh with an element size of $2.8 \mathrm{~mm}$, whereas for an element size of $1.5 \mathrm{~mm}$, the overestimation is only $30 \%$. Using quadratic elements (C3D10) the frequency is almost stable with a better estimation of the reference value of about $10 \%$. Note that the number of degrees of freedom and CPU times are almost identical for the mesh with quadratic elements of $2.8 \mathrm{~mm}$ or for linear elements of $1.5 \mathrm{~mm}$. Nevertheless, an overestimation of $12 \%$ of the reference value is observed using the quadratic elements, while it is only 30\% for the linear elements. This difference can be explained by the limited number of deformation modes for the linear elements, making them stiffer than their
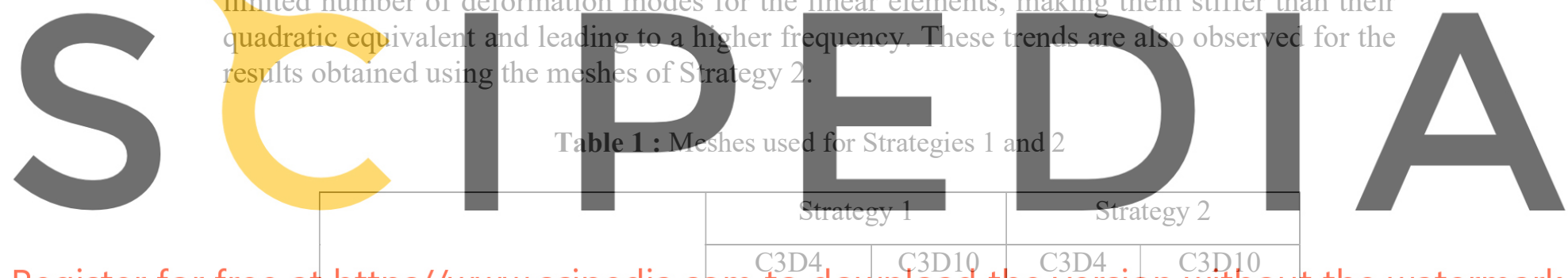

\begin{tabular}{|c|c|c|c|c|c|}
\hline \multirow{3}{*}{$\begin{array}{l}\text { M1 element } \\
\text { size } 2.8 \mathrm{~mm}\end{array}$} & nb elements & \multicolumn{2}{|c|}{117677} & \multicolumn{2}{|c|}{170163} \\
\hline & $\mathrm{nb}$ dof** & 89667 & 593361 & 127383 & 849384 \\
\hline & CPU time (s) & 19.4 & 183.6 & 24.9 & 258.8 \\
\hline \multirow{3}{*}{$\begin{array}{l}\text { M2 element } \\
\text { size } 1.5 \mathrm{~mm}\end{array}$} & nb elements & \multicolumn{2}{|c|}{726296} & \multicolumn{2}{|c|}{767590} \\
\hline & nb dof & 474984 & 3354123 & 506148 & 3564405 \\
\hline & CPU time (s) & 152.6 & 5424 & 158.3 & 5783 \\
\hline
\end{tabular}

C3D4 and C3D10 are linear and quadratic tetrahedral elements, respectively. ${ }^{* *} \mathrm{Nb}$ dof: Number of degrees of freedom.

The geometry obtained with Strategy 3 is comparable to the one generated by CAD software and allows the use of different types of elements to be tested, thus reducing CPU time and improving the accuracy of the results. The hexahedral solid elements are suitable for discretizing the parallelepipedal shaped plate. Note that prismatic triangular elements are used 
to represent the stiffener junctions. The first two columns of Table 3 show 5 meshes ranked from coarsest to finest using linear (hexahedral elements C3D8 and triangular prismatic elements C3D6) or quadratic (hexahedral elements C3D20 and triangular prismatic elements C3D15) elements with one element through the thickness. These five meshes are tested to study the convergence of the meshes. These regular meshes are generated by carrying out a prior adapted partitioning of the piece for $35 \mathrm{~min} 30 \mathrm{~s}$. For quadratic elements, results converge rapidly from the mesh with an element size of $30 \mathrm{~mm}$, whereas for their linear counterpart, equivalent results are only achieved considering an element size of $6 \mathrm{~mm}$. The CPU time for each computation is less than two seconds which is very reasonable.

Table 2 : Meshes used for Strategy 3

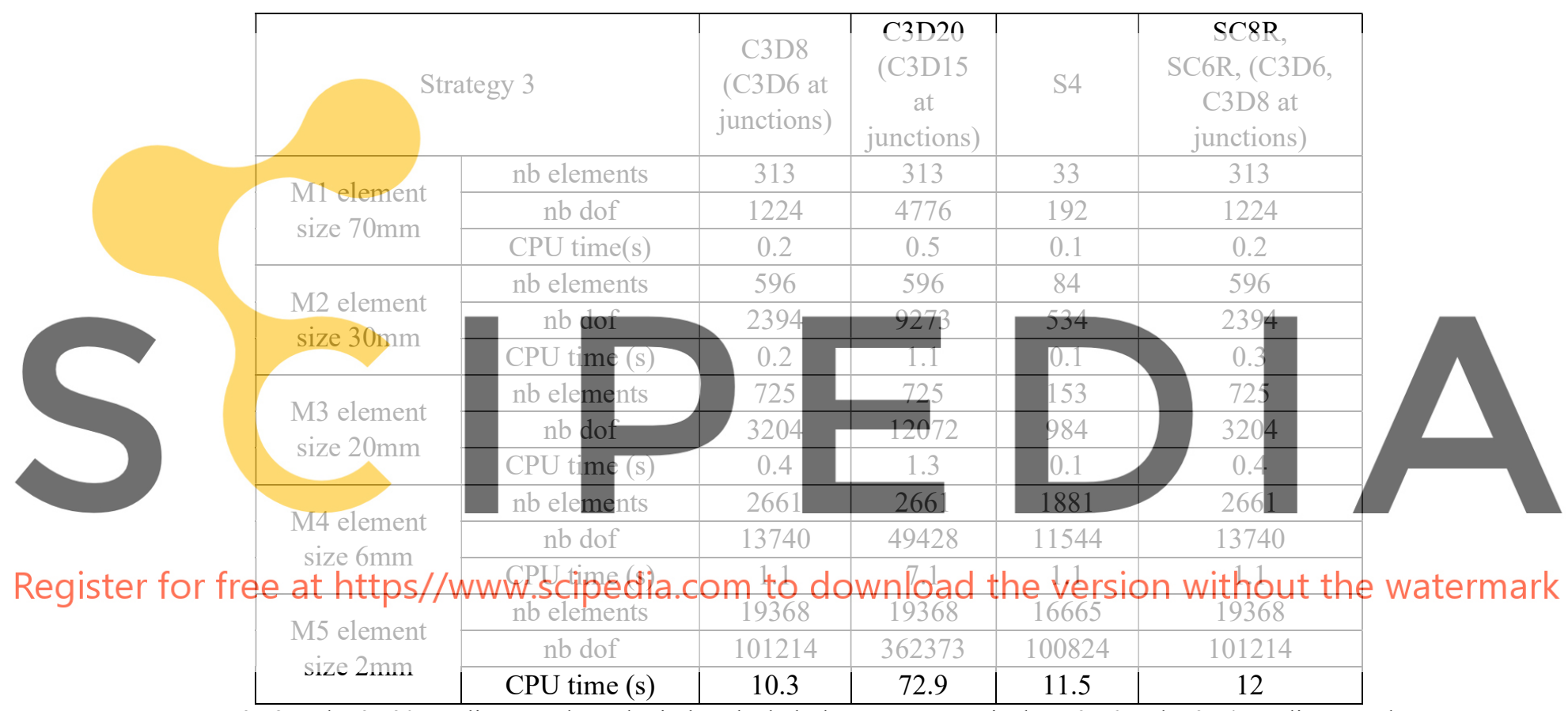

C3D8 and C3D20 are linear and quadratic hexahedral elements, respectively. C3D6 and C3D4 are linear and quadratic triangular prismatic elements, respectively. SC6R and SC8R are prismatic and hexahedral solid-shell elements. S4 is shell elements.

Other types of elements are available in Abaqus Software [16]. As the part is thin, it is possible to use shell elements [13] to quickly obtain accurate results. For example, the shell element (S4) can model the deformation of thin structures in membrane or bending. However, it is necessary to transform the geometry of the solid into a surface model by extracting its mid surface. In this case, the duration of this task is $5 \mathrm{~min}$ in Abaqus. Figure 6 clearly shows a rapid convergence of frequencies using 1,000 degrees of freedom, unlike using C3D20/C3D15 elements where convergence is using more than 10,000 degrees of freedom.

Finally, a combination of linear hexahedral (SC8R) and triangular prismatic (SC6R) solidshell elements with only displacement degrees of freedom at the corner nodes can also be used to obtain results comparable to shell elements in a minimum of time and without having 
to transform the geometry. See [14-15] as example of the formulation of solid-shell elements. To properly use solid-shell elements it is necessary to identify the thickness direction (or opposite external surfaces. A layer of an element is required to describe the thin wall of the part by indicating the free opposing surfaces. Thus, these elements cannot be used in the junctions of the part where the thin walls meet. In this case, linear hexahedral (C3D8) and prismatic (C3D6) solid elements must be used as they ensure compatibility with the solid shell elements SC8R or SC6R. Although the number of degrees of freedom is identical to those for meshes using C3D8/C3D6 elements, the results in Figure 6 show that the frequency has already converged using 1000 degrees of freedom, whereas using linear hexahedral elements the convergence is reached using more than 10,000 degrees of freedom. In this case, the use of solid-shell elements is the best compromise between accuracy of the results and the reasonable CPU times.

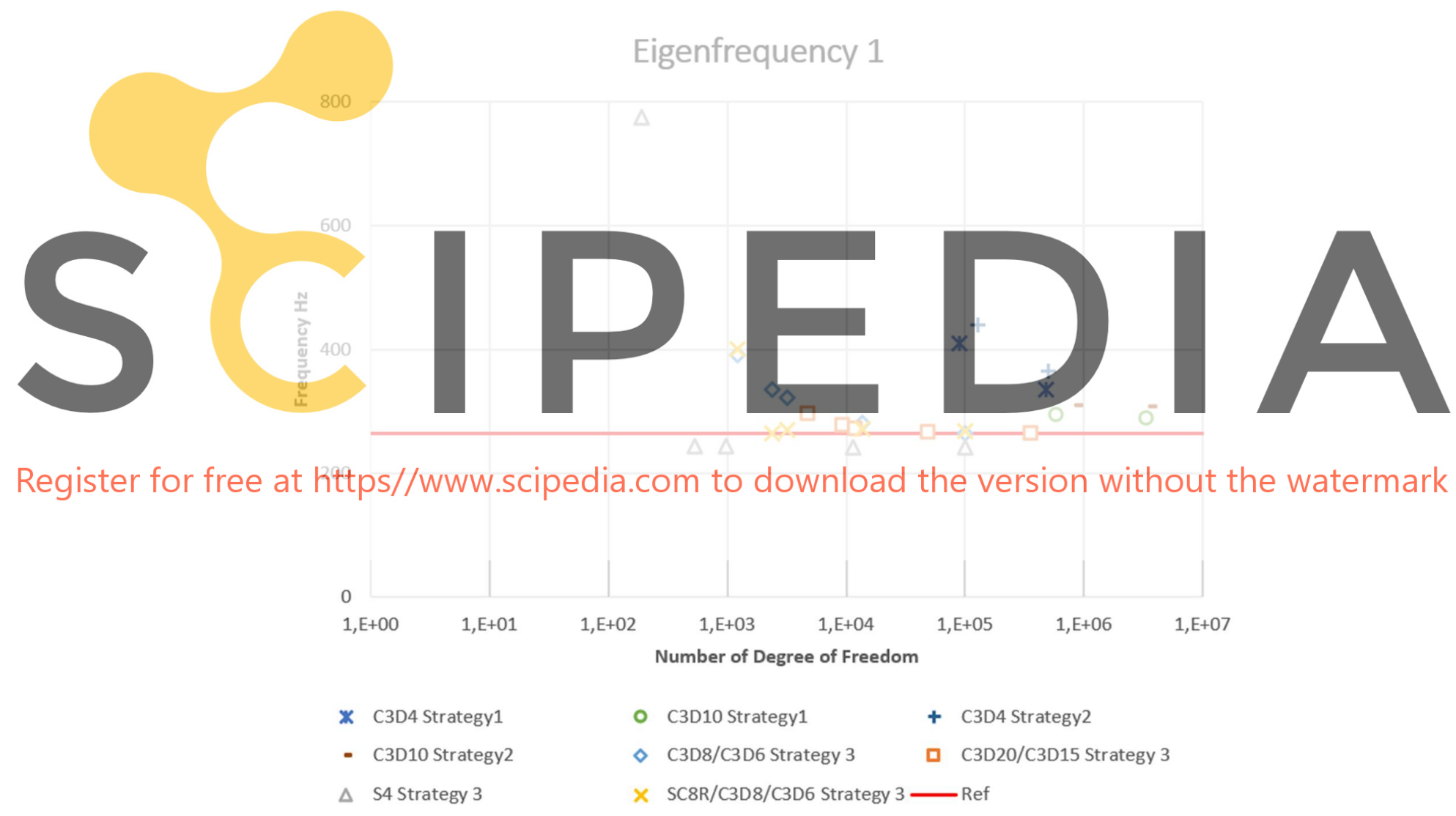

Figure 6 : First eigenfrequency computed with different meshes. The Reference result (Ref) has been obtained using C3D20/C3D15 with an element size of 2mm, 57452 elements (3 elements in thickness), 819255 dof. 


\section{CONCLUSIONS}

Strategies 1 and 2 require a significant amount of time (2H30) to capture as much data as possible during the scanning phase in order to be close to the original geometry. However, the reconstruction phase is relatively fast because it does not require specific skills. Nevertheless, the geometries obtained limit the size and choice of the type of finite element to be used for structural analysis. The CPU time is of the order of a few hours to obtain accurate results. Strategy 3, requires advanced skills in the software dedicated to reverse engineering. In addition, if the geometry is complex, the duration of the reconstruction will increase considerably. Nevertheless, this strategy allows the choice of different types of elements, reducing considerably the CPU time. We recommend Strategies 1 and 2 for preliminary studies that do not require precise calculations and expertise for reconstruction. Both strategies can be carried out while waiting for a more complete reconstruction with Strategy 3 to conduct an accurate FEM analysis.

\section{REFERENCES}

[1] Castellazzi, G., D'Altri, A.M., Bitelli, G., Selvaggi, I. and Lambertini, A. From laser scanning to finite element analysis of complex buildings by using a semi-automatic procedure. Sensors. (2015) 15(8):18360-80.
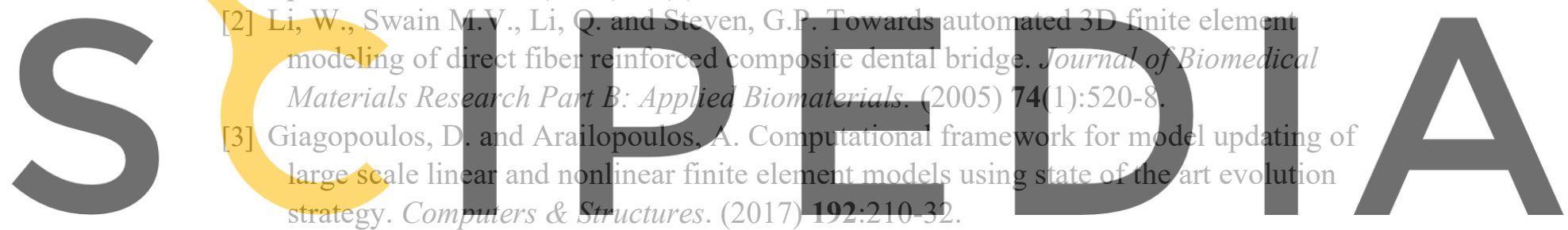

[4] Schneider, M., Friebe, H. and Galanulis, K. Validation and optimization of numerical

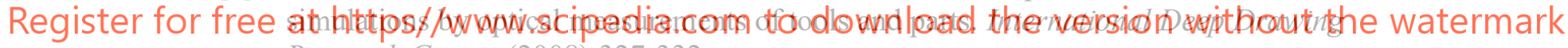
Research Group, (2008):327-332.

[5] Gentilini, I. and Shimada, K. Predicting and evaluating the post-assembly shape of thinwalled components via 3D laser digitization and FEA simulation of the assembly process. Computer-aided design, (2011) 43(3):316-328.

[6] Greco, F., Coox, L., Maurin, F., Balla, V. K., Deckers, E. and Desmet, W. Reverse engineering of deep drawn components with an isogeometric framework. Computers \& Structures. (2018). 201:15-25.

[7] Varady, T., Martin, R. R. and Cox, J. Reverse engineering of geometric models - an introduction. Computer-aided design. (1997) 29(4):255-268.

[8] Chang, K. H., \& Chen, C. 3D shape engineering and design parameterization. ComputerAided Design and Applications. (2011) 8(5):81-692.

[9] Buonamici, F., Carfagni, M., Furferi, R., Governi, L., Lapini, A., and Volpe, Y. Reverse engineering modeling methods and tools: a survey. Computer-Aided Design and Applications. (2018) 15(3):443-464.

[10] BREUCKMANN http://www.breuckmann.com

[11] GEOMAGIC http://www.geomagic.com 
[12] Eck, M. and Hoppe, H. Automatic reconstruction of B-spline surfaces of arbitrary topological type. In Proceedings of the 23rd annual conference on Computer graphics and interactive techniques. (1996):325-334.

[13] Batoz, J. L. and Dhatt, G. Modélisation des structures par éléments finis: Solides élastiques. Presses Université Laval (1990).

[14] Hannachi, M., Naceur, H. and Batoz, J. L. Continuum Based Solid-Shell Element Modeling for the Optimization of Composite Multilayered Structures. International Review of Mechanical Engineering. (2007) 1(4):150-163.

[15] Naceur, H., Shiri, S., Coutellier, D. and Batoz, J. L. On the modeling and design of composite multilayered structures using solid-shell finite element model. Finite Elements in Analysis and Design. (2013) 70:1-14.

[16] ABAQUS https://www.3ds.com/products-services/simulia/products/abaqus/ 